Open Access

\title{
A Japanese prospective multi-institutional feasibility study on accelerated partial breast irradiation using interstitial brachytherapy: treatment planning and quality assurance
}

Yuki Otani ${ }^{*}$, Takayuki Nose ${ }^{2}$, Takushi Dokiya ${ }^{3}$, Toshiaki Saeki ${ }^{4}$, Yu Kumazaki ${ }^{5}$, Shuuji Asahi ${ }^{6}$, Iwao Tsukiyama ${ }^{7}$ Ichirou Fukuda ${ }^{8}$, Hiroshi Sekine ${ }^{9}$, Naoto Shikama ${ }^{5}$, Takao Takahashi ${ }^{4}$, Ken Yoshida ${ }^{10}$, Tadayuki Kotsuma ${ }^{11}$, Norikazu Masuda $^{12}$, Eisaku Yoden ${ }^{13}$, Kazutaka Nakashima ${ }^{14}$, Taisei Matsumura ${ }^{15}$, Shino Nakagawa ${ }^{16}$, Seiji Tachiiri ${ }^{17}$, Yoshio Moriguchi ${ }^{18}$, Jun Itami ${ }^{19}$ and Masahiko Oguchi ${ }^{20}$

\section{Abstract}

Background: In Japan, breast-conserving surgery with closed cavity has generally been performed for breast cancer patients, and accelerated partial breast irradiation (APBI) is considered difficult because Asian females generally have smaller breast sizes than Western females. Therefore, common identification of target and treatment plan method in APBI is required. A prospective multicenter study was conducted in Japan to determine institutional compliance with APBI using high-dose-rate interstitial brachytherapy (ISBT) designed for Japanese female patients.

Methods: For this study, 46 patients were recruited at eight institutions from January 2009 to December 2011. The reproducibility of the ISBT-APBI plan was evaluated using three criteria: (1) minimum clinical target volume dose with a clip dose $\geq 6 \mathrm{~Gy} /$ fraction, (2) irradiated volume constraint of $40-150 \mathrm{~cm}^{3}$, and (3) uniformity of dose distribution, expressed as the dose non-uniformity ratio $(\mathrm{DNR}, \mathrm{V} 150 / \mathrm{N} 100)<0.35$. The ISBT-APBI plan for each patient was considered reproducible when all three criteria were met. When the number of non-reproducible patients was $\leq$ 4 at study completion, APBI at this institution was considered statistically reproducible.

Results: Half of the patients (52\%) had a small bra size (A/B cup). The mean values of the dose-constrained parameters were as follows: Vref, $117 \mathrm{~cm}^{3}$ (range, 40-282), DNR, 0.30 (range, 0.22-0.51), and clip dose, 784 cGy (range, 469-3146). A total of 43/46 treatment plans were judged to be compliant and ISBT-APBI was concluded to be reproducible.

Conclusions: This study showed that multi-institutional ISBT-APBI treatment plan was reproducible for small breast patient with closed cavity.

Keywords: APBI, HDR, Brachytherapy

\footnotetext{
* Correspondence: y.otani@radonc.med.osaka-u.ac.jp

1Department of Radiation Oncology, Osaka University Graduate School of

Medicine, 2-2 Yamadaoka, Suitashi, Osaka 565-0871, Japan

Full list of author information is available at the end of the article
} 


\section{Background}

In Japan, the incidence and mortality rate of breast cancer is growing rapidly, and the annual number of cases is expected to rise above 50,000 by 2020 [1]. Furthermore, the peak age for breast cancer in Japan is 10 years younger than that in Europe and USA, and the disease rate in 45-50-year-old females is high [2].

Breast-conserving surgery followed by postoperative radiotherapy is the standard of care for early-stage breast carcinoma [3, 4]. Generally, postoperative irradiation by whole-breast irradiation (WBI) reduces the rate of ipsilateral breast recurrence by one-third $[4,5]$. However, studies have also reported that WBI prevents recurrence only near the tumor bed $[3,4,6]$. Moreover, WBI raises the mortality rate by increasing the risk of cardiovascular adverse events [5]. Thus, localized irradiation of the tumor bed is preferable for patients at a low risk of recurrence.

Accelerated partial breast irradiation brachytherapy (APBI) delivers radiation near the tumor bed over a short period of time [7-11]. In Europe and the USA, successful phase I/II clinical trials were completed in the 1990s, and large-scale phase III clinical trials are currently underway $[8,10]$. In contrast, APBI is rarely used in Japan. One reason for this is the difficulty in target identification, caused by oncoplastic surgery, which is generally adopted in Japan. Moreover, APBI is considered difficult because Asian females generally have smaller breast sizes than Western females.

To promote APBI using interstitial brachytherapy, developing target identification consensus and reproducibility of this treatment among institutions should be first confirmed prospectively. This study presents the creative method of clinical target volume (CTV) and dose limitation for Asian females. Then, the reproducibility of interstitial brachytherapy (ISBT)-APBI is evaluated in breast cancer patients on multi-institutional feasibility clinical trial in Japan.

\section{Methods}

\section{Patient cohort}

A multi-institutional clinical trial was conducted from October 2009 to December 2011 at eight institutions in Japan. In total, 46 female patients with breast cancer were recruited prospectively for this study to receive adjuvant radiotherapy by ISBT-APBI alone after breastconserving surgery.

The Institutional Review Board (IRB) of each institution approved this study. Written informed consent for data acquisition was obtained from each patient.

\section{Surgery}

All patients underwent breast-conserving surgery, defined as resection of the primary tumor with $>0 \mathrm{~cm}$ of microscopic free margin. During surgery, the walls of the excision cavity were marked with at least four clips on the superior, inferior, medial, and lateral margins of the tumor bed. The brachytherapy applicators were inserted within 2 months after the breast-conserving surgery. All needles were inserted under image guidance by computed tomography (CT) or ultrasonography (US). More two-plane implants were made. The needles were replaced with flexible catheters and fixed with buttons. If applicator placement was judged insufficient during treatment planning, additional insertions were made.

\section{Brachytherapy}

After applicator implantation, CT data were acquired with the patient in a supine position. CT slice thickness was < $3.0 \mathrm{~mm}$. CT-based treatment planning was performed with the aid of Oncentra or PLATO (Nucletron BV, Veenendaal, The Netherlands). The high-dose-rate (HDR)ISBT protocol was performed with microSelectron-HDR V2 using ${ }^{192}$ Ir as the source for monotherapy.

The clinical target volume was defined by drawing 3$\mathrm{cm}$ diameter circles around each clip, and was considered to be the domain to which they were clinically connected (Fig. 1). The breast muscle layer and tissue of up to $5 \mathrm{~mm}$ depth from the skin surface were excluded. In addition, non-mammary gland tissue domain falling under CTV, identified by a consensus of the radiation oncologist and the breast surgeon, was excluded from CTV. The initial dose distribution was made using the Paris dosimetry system, which was modified by manual geometrical optimization when the skin dose was reduced. The brachytherapy was initiated the next day after applicator placement. A total dose of 36 Gy (six fractions of $6 \mathrm{~Gy}$ ) was delivered as two fractions per day with an interval of $6 \mathrm{~h}$ between the two fractions.

\section{Dose specification}

Compliance of each institution to the APBI protocol was assessed using three criteria:

1. Clip dose: The surgical clips in CTV were irradiated by $>100 \%$ of the isodose lines. A clip outside a mammary gland was excluded as an object. No exception was permitted.

2. Reference volume (Vref): The irradiated volume receiving $>100 \%$ of the prescription dose was $40-$ $150 \mathrm{~cm}^{3}$. An exception was made for specific conditions (e.g., larger breast) under the approval of the study office and data center.

3. Dose non-uniformity ratio (DNR): The ratio was defined as the irradiated volume that received $\geq 1.5$ times the reference dose over the volume that received $\geq 1.0$ times the reference dose $(\mathrm{DNR}=$ V1.5ref/Vref). For all patients, DNR must be $<0.35$, with no exception. 


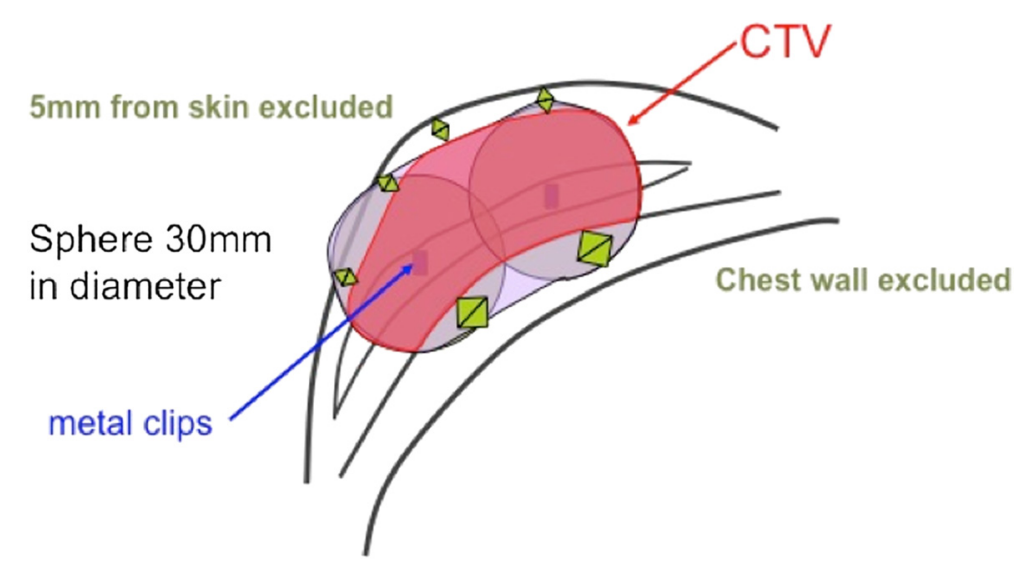

Fig. 1 Clinical target volume (CTV) defines method

Compliance with the treatment plan was acknowledged when all three criteria were within the reference range.

\section{Quality assurance}

To improve the quality of this study, workshops, rapid review, and Interim analysis were established. Quality assurance was implemented according to Radiation Therapy Oncology Group (RTOG) procedures [12]. Moreover, the data center predicted the total irradiation time from the Vref and source activity.

\section{Workshops}

Three workshops were conducted to homogenize treatments between institutions. A radiation oncologist, a breast surgeon, a medical physicist, and a radiation technologist from each institution participated in all three workshops. The participants discussed the process of applicator insertion and the treatment plan. Moreover, each institution was instructed to submit their calculation of the benchmark treatment plan using uniform sample patient data, and the submitted data were verified at the data center.

Table 1 Patient demographics

\begin{tabular}{lll}
\hline Variable & Mean \pm SD (Range) & N (\%) \\
\hline Weight $(\mathrm{kg})$ & $60 \pm 11(43-96)$ & \\
Height $(\mathrm{cm})$ & $155 \pm 6(140-170)$ & \\
Body Mass Index & $25.0 \pm 4.8(18.4-41.6)$ & \\
Number of catheters (n) & $15 \pm 3(8-21)$ & \\
Breast excision weight (g) & $82 \pm 44(25-234)$ & \\
Volume of CTV $\left(\mathrm{cm}^{3}\right)$ & $67 \pm 29(26-133)$ & $10(22)$ \\
Bra size & & $14(30)$ \\
A cup & & $11(24)$ \\
B cup & & $11(24)$ \\
C cup & & \\
$\geq$ D cup & & \\
\hline
\end{tabular}

\section{Rapid review}

Each institution was required to fax a case report form (CRF) to the data center at least $24 \mathrm{~h}$ before the onset of treatment. The treatment plan summary contained no patient identifier. If there was any doubt, the data center queried the relevant institution and consulted with the study office. The data center and study office verified the following parameters: applicator length, offset value, clip dose, Vref, DNR, maximum skin dose, total treatment time, and source strength.

\section{Interim and final analyses}

The number of patients required for the statistical analysis was determined on the basis of the optimal design of Simon [13]. Interim analysis was performed with data from 19 patients, and this clinical trial was planned to be stopped when non-compliance with the plan was confirmed for $>3$ patients. Final analysis was performed with data from 46 patients. The ISBT-APBI plan was defined as reproducible if non-compliance with the plan was confirmed for $<5$ patients. The electronic data on

Table 2 Index parameters of the treatment plan

\begin{tabular}{ll}
\hline Variable & Mean \pm SD (Range) \\
\hline Vref $\left(\mathrm{cm}^{3}\right)$ & $117 \pm 56(40-282)$ \\
DNR & $0.30 \pm 0.05(0.22-0.51)$ \\
Clip dose (cGy) & $784 \pm 224(469-3146)$ \\
Max skin dose (cGy) & $521 \pm 73(223-607)$ \\
CTV V1.5ref $\left(\mathrm{cm}^{3}\right)$ & $39 \pm 18(12-98)$ \\
CTV V2.0ref $\left(\mathrm{cm}^{3}\right)$ & $15 \pm 9(5-58)$ \\
CTV V100 (cm $\left.{ }^{3}\right)$ & $63 \pm 27(25-129)$ \\
CTV V90 (cm $\left.{ }^{3}\right)$ & $63 \pm 28(26-131)$ \\
CTV D100 (cGy) & $565 \pm 125(146-624)$ \\
CTV D90 (cGy) & $680 \pm 97(225-731)$ \\
CTV Mean dose (cGy) & $927 \pm 80(760-1092)$ \\
Conformity Index & $2.07 \pm 0.84(1.07-4.45)$ \\
\hline
\end{tabular}



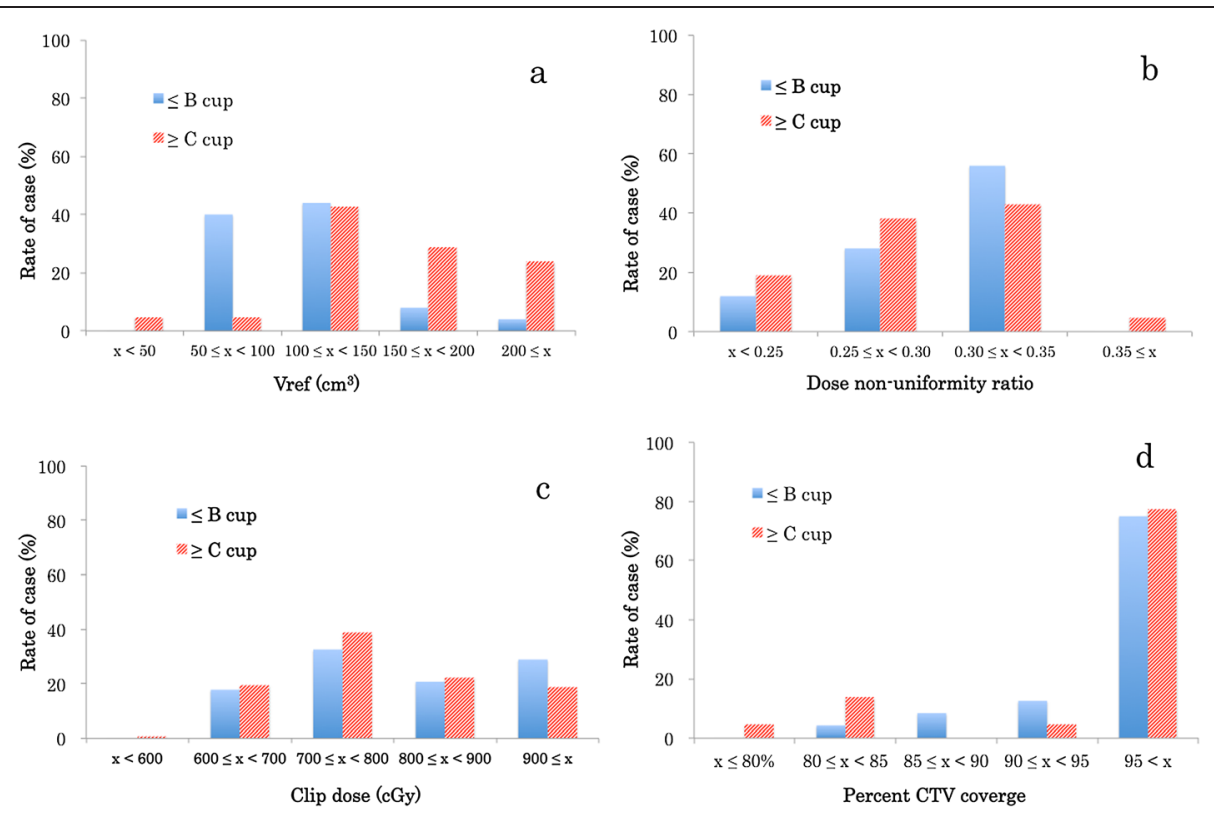

Fig. 2 Frequency distribution of the index parameters of the ISBT-APBI treatment plan. a Reference volume (Vref). b Dose non-uniformity ratio (DNR). c Clip dose. $\mathbf{d}$ Percent CTV coverage

the treatment plan from each institution was imported to Oncentra at the data center anonymously. The data center and study office reviewed all treatment plans.

\section{Results}

\section{Patient demographics and treatment plan index}

The patient demographics are shown in Table 1. The patients were subjected to postoperative implantation by closed cavity (after breast-conserving surgery, $n=45$ ) or perioperative implantation (during intra-breast-conserving surgery, $n=1)$. There were 24 patients $(52 \%)$ with a small bra size (A or B cup). Table 2 shows the index parameters of the treatment plan. The mean values of the doseconstrained parameters were as follows: Vref, $117 \mathrm{~cm}^{3}$ (range, 40-282), DNR, 0.30 (range, 0.22-0.51), and clip dose, 784 cGy (range, 469-3146). Fig. 2 shows the normal frequency distributions for Vref, DNR, clip dose, and percent CTV coverage among patients. The relationship among

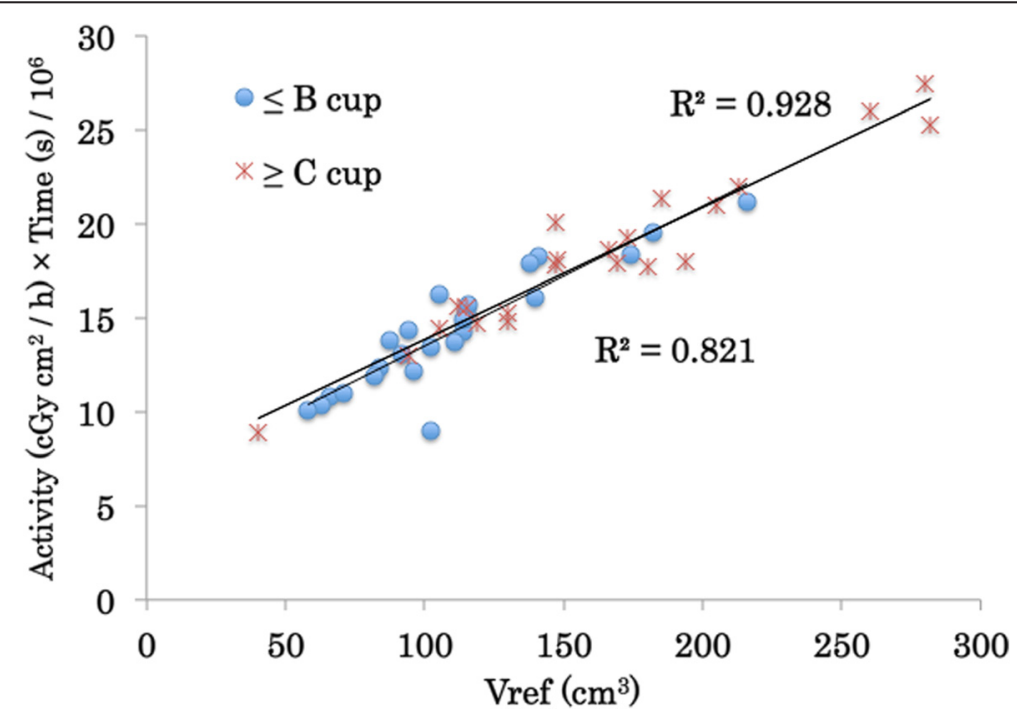

Fig. 3 The relationship among activity, time and Vref. Activity is the activity the high-dose-rate source, time (s) is the total irradiation time, and Vref is the reference volume 
source activity, total irradiation time, and Vref are shown in Fig. 3. The correlation factors for in small bra size and large bra size patients were 0.821 and 0.928 , respectively.

\section{Validation of the reference volume}

The data center and study office evaluated the adequacy of the Vref values that were outside the $40-150 \mathrm{~cm}^{3}$ range. In total, 14 patients had a Vref above the upper limit, with a median of $191 \mathrm{~cm}^{3}$ (range, 169-287). The patients with a Vref of $>150 \mathrm{~cm}^{3}$ had a median body mass index (BMI) of 28.3 (range, 25.5-41.6) and a median excision weight of $110 \mathrm{~g}$ (range, 60-234). In contrast, those with a Vref of $\leq$ $150 \mathrm{~cm}^{3}$ had a median BMI of 22.5 (range, 18.4-30.8) and a median excision weight of $61 \mathrm{~g}$ (range, 25-100). All exceeding Vref values were determined to be appropriate.

\section{Interim and final analyses}

The interim analysis identified two non-compliant treatment plans. The analysis report was submitted to the safety monitoring committee, and clinical trial continuation was approved. In the final analysis, one more treatment plan was judged non-compliant with the protocol.

In case \#1, the DNR value was 0.51 , which was not within the acceptable limit $(<0.35)$. This discrepancy was due to the large size of the patient's breast (I cup). The target was larger than expected because the cavity was filled with fluid, which required several applicators. However, the patient refused the insertion of additional applicators. In case \#2, the CTV creative method was not in accordance with the protocol. Not all $3-\mathrm{cm}$ spheres created around the clips were connected. However, the dose distribution was connected, which was judged to be clinically satisfactory. In case \#3, the one clip dose was 469 cGy, less than the reference value and wrong clip dose value was written in CRF. Thus, a total of $43 / 46$ treatment plans were judged to be compliant and ISBT-APBI was concluded to be reproducible.
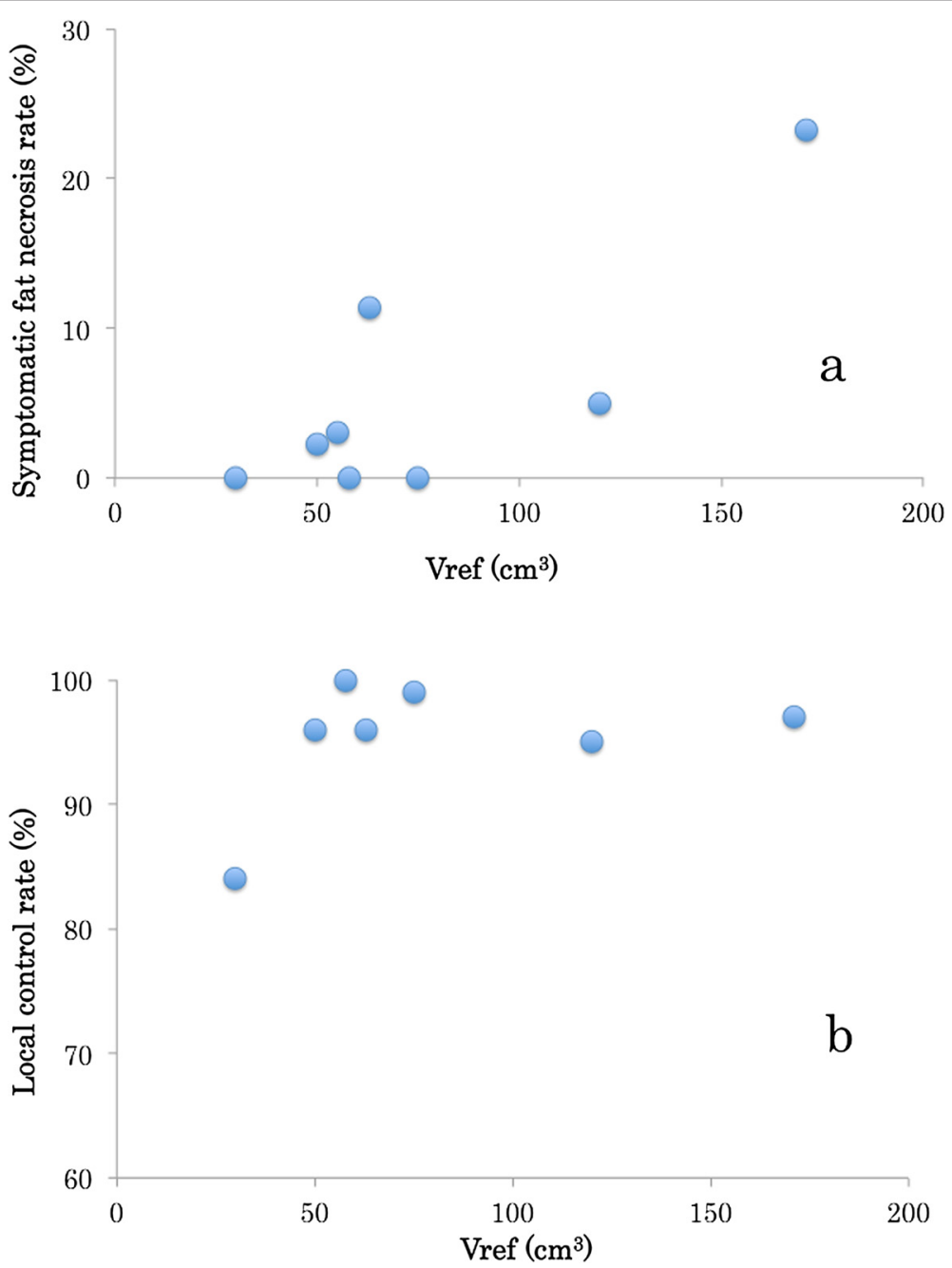

Fig. 4 The relationship between irradiated volume, fat necrosis, and local control rate. a fat necrosis and Vref. b relationship local control rate and Vref 


\section{Discussion}

This study was designed to evaluate the reproducibility of ISBT-APBI for breast cancer patients among Japanese institutions. To our knowledge, this is the first reported ISBT-APBI multi-institutional clinical trial in Asia. In this study, ISBT-APBI was compliant for $43 / 46$ patients. Although ISBT involves multiple manual procedures, standardization of the treatment plan is possible through comprehensive webinars. Two cases (case \#2 and \#3) deviated from the protocol due to a human error detected in CRF. Thus, careful review of the electronic data on the treatment plan is essential to secure the quality of a multicenter clinical trial.

Based on preceding literature, two indices (Vref and DNR) of a brachytherapy plan are found to be important for local control and sequel. Figure 4 shows the relationship between irradiated volume, fat necrosis, and local control rate which have been reported by some authors [11, 14-21]. Wazer et al. reported that Vref, V1.5xref, and V2.0ref correlated with fat necrosis [16, 22]. Ott et al. concluded that irradiation volume correlated significantly with side-effects and local control, and a suitable upper limit of Vref was $150-180 \mathrm{~cm}^{3}$ for a closed cavity [9]. In contrast, Perera et al. reported a local control ratio of $84 \%$ in an irradiated volume of $30 \mathrm{~cm}^{3}$ [17]. In literature from Germany and Austria, the Vref range was $48-84 \mathrm{~cm}^{3}[9,18-20]$. The protocol of the Phase III multi-institutional clinical trial of the Groupe Européen de Curiethérapie-European Society for Therapeutic Radiology and Oncology (GEC-ESTRO) adopted a Vref range of $40-150 \mathrm{~cm}^{3}$ with a DNR of $<0.35$ [10]. In USA, the rate of open cavity breast surgery and the radiation volume are larger than those in Europe and Japan. Thus, the reference indicators of The National Surgical Adjuvant Breast and Bowel Project (NSABP)/RTOG $0413[8,10]$ may not apply to other continents.

Most patients with excessive Vref had a pyknic body type and required large-weight excisions. Moreover, six (13\%) patients had a BMI of $>30$, corresponding to a $3.2 \%$ deviation (Organization for Economic Co-operation and Development Health data 2009 [23]) from the general Japanese female population. There is a possibility that the APBI plan deliberately selected large-breast patients, because most Japanese females have small breasts and insertion of the applicator is difficult. This ISBT-APBI plan provided a better coverage of the target area, because a median $98 \%$ of CTV received $100 \%$ of the prescription dose, compared to $96 \%$ of the single-institutional study by Das et al. [24].

The quality assurance methods of HDR treatment planning have been reported [24-26]. One of a quick, easy way of checking is a method to infer the total irradiation time from the source activity and the Vref. According to our results, there is low correlation among the Vref, the source activity and the total treatment time in the small bra-size treatment plan compared with that in the large bra-size treatment plan (Fig. 3). For small bra size patients, applicator insertion is difficult and the applicator spacing tends to be irregular and narrow. Moreover, each point source stop time in the small bra-size patient treatment plan is short compared with that in the large bra-size patient. The Vref increases exponentially with increasing source stop time because the dose distribution expands concentrically. Therefore, the method of inferring total irradiation time from the source activity and Vref is difficult to adjust to patients with small breasts.

\section{Conclusions}

This prospective study defined an ISBT-APBI treatment plan to be clinically feasible for most Asian females with a closed cavity and small breast size. Furthermore, the treatment plan was reproducible between institutions, despite various levels of skills and experience with the technique.

\section{Competing interests \\ The authors declare that they have no competing interests.}

\section{Authors' contributions}

YO was responsible for writing and editing the manuscript, and extensive data analysis. TN made substantial contributions to conception and design. TD and MO were responsible for managing the clinical trial. All authors were responsible for patient accrual and management. All authors read and approved the final manuscript.

\section{Acknowledgements}

This study was supported in part by a Grant-in-Aid for Cancer Research from the Ministry of Health, Labour and Welfare of the Government of Japan (17-10 and 21-8-2) and MEXT KAKENHI Grant Number 26860401.

\section{Author details}

${ }^{1}$ Department of Radiation Oncology, Osaka University Graduate School of Medicine, 2-2 Yamadaoka, Suitashi, Osaka 565-0871, Japan. ${ }^{2}$ Department of Radiation Oncology, Nippon Medical School, Tamanagayama Hospital, Tama, Tokyo, Japan. ${ }^{3}$ Department of Radiation Oncology, Kyoundo Hospital, Sasaki-Foundation, Chiyodaku, Tokyo, Japan. ${ }^{4}$ Department of Breast Oncology, Saitama Medical University, International Medical Center, Hidaka, Saitama, Japan. ${ }^{5}$ Department of Radiation Oncology, Saitama Medical University, International Medical Center, Hidaka, Saitama, Japan. ${ }^{6}$ Department of Surgery, Aidu Chuo Hospital, Aiduwakamatsu, Fukushima, Japan. 'Head of research institute for radiotherapy Southern TOHOKU Research Institute for Neuroscience Southern TOHOKU General Hospital, Koriyama, Fukushima, Japan. ${ }^{8}$ Department of Radiation Oncology, National Hospital Organization Disaster Medical Center, Tachikawa, Tokyo, Japan. ${ }^{9}$ Department of Radiology and Radiotherapy, The Jikei University, Daisan Hospital, Komae, Tokyo, Japan. ${ }^{10}$ Department of Radiation Oncology, Osaka Medical College, Takatsuki, Osaka, Japan. ${ }^{11}$ Department of Radiation Oncology, National Hospital Organization Osaka National Hospital, Osaka, Japan. ${ }^{12}$ Department of Surgery, Breast Oncology, National Hospital Organization Osaka National Hospital, Osaka, Japan. ${ }^{13}$ Department of Radiation Oncology, Kawasaki Medical School, Kurashiki, Okayama, Japan. ${ }^{14}$ Department of General Surgery, Kawasaki Medical School, Kurashiki, Okayama, Japan. ${ }^{15}$ Department of Radiology, National Hospital Organization Kyushu Medical Center, Fukuoka, Fukuoka, Japan. ${ }^{16}$ Department of Breast Surgery, National Hospital Organization Kyushu Medical Center, Fukuoka, Fukuoka, Japan. ${ }^{17}$ Department of Radiation Oncology, Kyoto City Hospital, Kyoto, Kyoto, Japan. ${ }^{18}$ Department of Breast Oncology, Kyoto City Hospital, Kyoto, Kyoto, Japan. ${ }^{19}$ Department of Radiation Oncology, National Cancer Center Hospital, Tokyo, Japan. 
${ }^{20}$ Department of Radiation Oncology, Cancer Institute Hospital, the Japanese Foundation for Cancer Research, Tokyo, Japan.

Received: 19 December 2014 Accepted: 27 May 2015 Published online: 04 June 2015

\section{References}

1. Ohno Y, Nakamura T, Murata K, Tsukuma H, Ajiki W, Ooshima A. Prediction of cancer incidence in Japan: Projections up to 2020 based on the analyses using a Bayesian age-period-cohort model: Cancer Statistics. Shinohara Shuppan Tokyo. 2004;4(4):201-17.

2. Yoshimoto M, Tada K, Hori H, Morota A, Tanabe M, Nishimura S, et al. Improvement in the prognosis of Japanese breast cancer patients from 1946 to 2001-an institutional review. Jpn J Clin Oncol. 2004;34:457-62.

3. Veronesi U, Cascinelli N, Mariani L, Greco M, Saccozzi R, Luini A, et al. Twenty-year follow-up of a randomized study comparing breast-conserving surgery with radical mastectomy for early breast cancer. N Engl J Med. 2002;17:1227-32.

4. Fisher B, Anderson S, Bryant J, Margolese RG, Deutsch M, Fisher ER, et al. Twenty-year follow-up of randomized trial comparing total mastectomy, lumpectomy, and lumpectomy plus irradiation for the treatment of invasive breast cancer. N Engl J Med. 2002;17:1233-41.

5. Early Breast Cancer Trialists' Collaborative Group. Favourable and unfavourable effects on long-term survival of radiotherapy for early breast cancer: an overview of the randomised trials. Lancet. 2000;355:1757.

6. Dirbas FM, Jeffrey SS, Goffinet DR. The evolution of accelerated, partial breast irradiation as a potential treatment option for women with newly diagnosed breast cancer considering breast conservation. Cancer Biother Radiopharm. 2004;19:673-705.

7. Chen PY, Vicini FA, Benitez P, Kestin LL, Wallace M, Mitchell C, et al. Long-term cosmetic results and toxicity after accelerated partial-breast irradiation: a method of radiation delivery by interstitial brachytherapy for the treatment of early-stage breast carcinoma. Cancer. 2006;106:991-9.

8. NSABP protocol B-39/ RTOG protocol 0413. A randomized phase III study of conventional whole breast irradiation (WBI) versus partial breast irradiation (PBI) for women with stage 0,1 , II breast cancer. http://www.rtog.org/members/ protocols/0413/0413.pdf.

9. Ott OJ, Hildebrandt G, Pötter R, Hammer J, Lotter M, Resch A, et al. Accelerated partial breast irradiation with multi-catheter brachytherapy: Local control, side effects and cosmetic outcome for 274 patients. Results of the German-Austrian multi-centre trial. Radiother Oncol. 2007;82:281-6.

10. European brachytherapy breast cancer GEC-ESTRO working group. Phase III multicenter trial. Interstitial brachytherapy alone versus external beam radiation therapy after breast conserving surgery for low risk invasive carcinoma and low risk duct carcinoma in-situ (DCIS) of the female breast. http://www.apbi.uni-erlangen.de/index.html.

11. Polgár C, Sulyok Z, Fodor J, Orosz Z, Major T, Takácsi-Nagy Z, et al. Sole brachytherapy of the tumor bed after conservative surgery for $\mathrm{T} 1$ breast cancer: five-year results of a phase $\mathrm{H}-\mathrm{I}$ study and initial findings of a randomized phase III trial. J Surg Oncol. 2002;80:121-8.

12. Ibbott GS, Hanson WF, O'Meara E, Kuske RR, Arthur D, Rabinovitch R, et al. Dose specification and quality assurance of radiation therapy oncology group protocol 95-17; a cooperative group study of iridium-192 breast implants as sole therapy. Int J Radiat Oncol Biol Phys. 2007;69:1572-8.

13. Simon R. Optimal two-stage designs for phase II clinical trials. Control Clin Trials. 1989;10:1-10.

14. Nose T, Komoike Y, Yoshida K, Koizumi M, Motomura K, Kasugai T, et al. A pilot study of wider use of accelerated partial breast irradiation: Intraoperative margin-directed re-excision combined with sole high-doserate interstitial brachytherapy. Breast Cancer. 2006;13:289-99.

15. Polgár C, Fodor J, Major T, Németh G, Lövey K, Orosz Z, et al. Breastconserving treatment with partial or whole breast irradiation for low-risk invasive breast carcinoma- 5-year results of a randomized trlal. Int J Radiat Oncol Biol Phys. 2007;69:694-702.

16. Wazer DE, Lowther D, Boyle T, Ulin K, Neuschatz A, Ruthazer R, et al. Clinically evident fat necrosis in women treated with high-dose-rate brachytherapy alone for early-stage breast cancer. Int J Radiat Oncol Biol Phys. 2001;50:107-11.

17. Perera F, Yu E, Engel J, Holliday R, Scott L, Chisela F, et al. Patterns of breast recurrence in a pilot study of brachytherapy confined to the lumpectomy site for early breast cancer with six years' minimum follow-up. Int J Radiat Oncol Biol Phys. 2003;57:1239-46.
18. Ott OJ, Schulz-Wendtland R, Uter W, Pfahlberg A, Beckmann MW, Sauer R, et al. Fat necrosis after conserving surgery and interstitial brachytherapy and/or external-beam irradiation in women with breast cancer. Strahlenther Onkol. 2005;181:638-44.

19. Vicini F, Arthur D, Polgar C, Kuske R. Defining the efficacy of accelerated partial breast irradiation: The importance of proper patient selection, optimal quality assurance, and common sense. Int I Radiat Oncol Biol Phys. 2003;57:1210-3.

20. Ott OJ, Pötter R, Hammer J, Hildebrandt G, Lotter M, Resch A, et al. Accelerated partial breast irradiation with iridium-192 multicatheter PDR/ HDR brachytherapy. Preliminary results of the German-Austrian multicenter trial. Strahlenther Onkol. 2004;180:642-9.

21. Lövey K, Fodor J, Major T, Szabó E, Orosz Z, Sulyok Z, et al. Fat necrosis after partial-breast irradiartion with brachytherapy or electron irradiation versus standard whole-breast radiotherapy-4-year results of a randomized trial. Int J Radiat Oncol Biol Phys. 2007;69:724-31.

22. Wazer DE, Kaufman S, Cuttino L, DiPetrillo T, Arthur DW. Accelerated partia breast irradiation: an analysis of variables associated with late toxicity and long-term cosmetic outcome after high-dose-rate interstitial brachytherapy. Int J Radiat Oncol Biol Phys. 2006;64:489-95.

23. Organisation for Economic Co-operation and Development (OECD). OECD Health Data 2009: Statistics and Indicators for 30 Countries. Paris: Organisation for Economic Co-operation and Development (OECD); 2009.

24. Das RK, Patel R, Shah H, Odau H, Kuske RR. 3D CT-based high-dose-rate breast brachytherapy implants: treatment planning and quality assurance. Int J Radiat Oncol Biol Phys. 2004;59:1224-8.

25. Takahashi Y, Koizumi M, Sumida I, Isohashi F, Ogata T, Akino Y, et al. The usefulness of an independent patient-specific treatment planning verification method using a benchmark plan in high-dose-rate intracavitary brachytherapy for carcinoma of the uterine cervix. J Radiat Res. 2012;53(6):936-44.

26. Das RK, Bradley KA, Nelson IA, Patel R, Thomadsen BR. Quality assurance of treatment plans for interstitial and intracavitary high-dose-rate brachytherapy. Brachytherapy. 2006;5(1):56-60.

\section{Submit your next manuscript to BioMed Central and take full advantage of:}

- Convenient online submission

- Thorough peer review

- No space constraints or color figure charges

- Immediate publication on acceptance

- Inclusion in PubMed, CAS, Scopus and Google Scholar

- Research which is freely available for redistribution

Submit your manuscript at www.biomedcentral.com/submit
C BioMed Central 